\title{
Groups whose lattices of normal subgroups are factorial
}

\author{
A. Rajhi \\ Communicated by L. A. Kurdachenko
}

\begin{abstract}
A Bstract. We prove that the groups $G$ for which the lattice of normal subgroups $\mathcal{N}(G)$ is factorial are exactly the UND-groups, that is the groups for which every normal subgroup have a unique normal complement, with finite length.
\end{abstract}

\section{Introduction}

The link between the structure of a group and the structure of some lattices of it's subgroups constitutes an important domain of research in group theory. The topic has enjoyed a rapid development starting with the first half of the 20th century. Many classes of groups determined by different properties of partially ordered subsets of their subgroups (especially lattices of subgroups or more particulary lattices of normal subgroups) have been identified. We refer to Schmidt's book [7] for more information about this theory. In this paper, we consider the lattice $\mathcal{N}(G)$ of normal subgroups of an arbitrary group $G$ as an arithmetic object. It is not clear for the moment what we mean by this. In order to clarify our idea, let's take an example. Let $n \geqslant 2$ be an integer and $\mathcal{D}(n)$ the partially ordered set (ordered by divisibility) of divisor's of $n$. This partially ordered set (for short poset) is actually a lattice in which the meet $a \wedge b$ and the join $a \vee b$ of two elements $a, b \in \mathcal{D}(n)$ are respectively the latest common

2010 MSC: 20E99,06B99.

Key words and phrases: lattice of normal subgroups, semilattices, idempotent monoids, partial monoids. 
multiple and the greatest common divisor of $a$ and $b$. Note that $\mathcal{D}(n)$ is a bounded lattice (in fact it is a finite lattice) in which the initial element is 1 and the final element is $n$. Moreover, the atoms of $\mathcal{D}(n)$ (ie. the minimal elements of $\mathcal{D}(n) \backslash\{1\})$ are exactly the prime divisor's of $n$ and the coatoms of $\mathcal{D}(n)$ (ie. the maximal elements of $\mathcal{D}(n) \backslash\{n\}$ ) are exactly the divisor's $a$ of $n$ such that the number $n / a$ is a prime number. We will say that the lattice $\mathcal{D}(n)$ is factorial (respectively cofactorial) if every element $a \in \mathcal{D}(n) \backslash\{1\}$ (respectively $a \in \mathcal{D}(n) \backslash\{n\}$ ) can be expressed uniquely (up to permutation) as join (respectively meet) of a finite number of pairwise distinct atoms (respectively coatoms). It is not hard to see that $\mathcal{D}(n)$ is factorial (respectively cofactorial) if and only if $n$ is a squarefree integer. Now we can introduce this notion for different algebraic structures. For example, let $V$ be a finite dimensional vector space and $L(V)$ the lattice of it's subspaces Recall that the meet and the join of two subspaces $F_{1}$ and $F_{2}$ of $V$ are given by

$$
F_{1} \wedge F_{2}=F_{1} \cap F_{2} \quad \text { and } \quad F_{1} \vee F_{2}=F_{1}+F_{2} .
$$

Note that the atoms (respectively coatoms) of $L(V)$ are exactly the one dimensional (respectively one codimension) subspaces of $V$. It is an easy exercise in linear algebra to check that every subspace $F \in L(V) \backslash\{0\}$ (respectively $F \in L(V) \backslash\{V\}$ ) can be expressed as join (respectively meet) of a finite number of pairwise distinct atoms (respectively coatoms) but that such decomposition can not be unique. The lattice $L(V)$ of subspaces of $V$ is then atomic (respectively coatomic) but it is not factorial (respectively cofactorial). Let $G$ be an arbitrary group and $\mathcal{N}(G)$ it's lattice of normal subgroups. In this paper, we show that the lattice $\mathcal{N}(G)$ is atomic (respectively coatomic) if and only if $G$ is an ND-group, that is a group in which every normal subgroup have a normal complement, with finite length on normal subgroups and that the lattice $\mathcal{N}(G)$ is factorial (respectively cofactorial) if and only if $G$ is an UND-group, that is a group in which every normal subgroup have a unique normal complement, with finite length on normal subgroups.

\section{Preliminaries}

Recall that a join-semilattice is a partially ordered set $(L, \leqslant)$ in which the joint $x \vee y$ (the least upper bound) of any two elements exists. Similarly, a meet-semilattice is a partially ordered set $L$ in which the meet $x \wedge y$ (the greatest lower bound) of any two elements exists. If $L$ is a join-semilattice (respectively meet-semilattice), the zero element (respectively unit element) 
of $L$, if it exists, is the least element (respectively the greatest element) of $L$, we denote it by $0_{L}$ (respectively $1_{L}$ ). If there is no risk of confusion, we denote by 0 (respectively by 1 ) the zero element (respectively unit element) of a join-semilattice (respectively meet-semilattice) $L$. For simplicity, a joinsemilattice (respectively meet-semilattice) with zero element (respectively unit element) is called a $(\vee, 0)$-semilattice (respectively $(\wedge, 1)$-semilattice). Note that every commutative monoid $(M,+, 0)$ in which every element is idempotent, which we call a commutative idempotent monoid, endowed with its algebraic partial ordering defined by $x \leqslant y$ if and only if $x+y=y$, is a $(\vee, 0)$-semilattice and it is a $(\wedge, 1)$-semilattice if we endow $M$ by the reverse order. Conversely, every $(\vee, 0)$-semilattice (respectively $(\wedge, 1)$ semilattice) $L$ can be viewed as a commutative idempotent monoid in which the addition is given by $x+y=x \vee y$ (respectively $x+y=x \wedge y$ ), for all $x, y \in L$. In this paper, by abuse of notation we don't make any difference between $(\vee, 0)$-semilattices (respectively $(\wedge, 1)$-semilattices) and commutative idempotent monoids. Let $L$ be a $(\vee, 0)$-semilattice. An element $p \in L \backslash\{0\}$ is called an atom of $L$, if for all $x, y \in L$ with $x \neq y$, $p=x \vee y$ implies $x=0$ or $y=0$. Dually, if $L$ is a $(\wedge, 1)$-semilattice, an element $q \in L \backslash\{1\}$ is called an coatom of $L$, if for all $x, y \in L$ with $x \neq y$, $q=x \wedge y$ implies $x=1$ or $y=1$.

Definition 1. Let $L$ be a $(\vee, 0)$-semilattice.

a) We say that $L$ is atomic if every nonzero element of $L$ is join of a finite number of atoms, that is for every $x \in L \backslash\{0\}$ there exist atoms $p_{1}, \ldots, p_{n}$ of $L$ such that $x=p_{1} \vee \cdots \vee p_{n}$.

b) We say that $L$ is factorial if it is atomic and if for every pairwise distinct atoms $p_{1}, \ldots, p_{n} \in L$ and for every pairwise distinct atoms $q_{1}, \ldots, q_{m} \in L$, if $p_{1} \vee \cdots \vee p_{n}=q_{1} \vee \cdots \vee q_{m}$, then $n=m$ and there is a permutation $\sigma \in S_{n}$ such that, for every $i \in\{1, \ldots, n\}$, $q_{i}=p_{\sigma(i)}$

We define dually coatomic and cofactorial $(\wedge, 1)$-semilattices. Let $(L, \wedge, \vee)$ be a bounded lattice in which the zero element is denoted by 0 and the unit element is denoted by 1 . We write $a \prec b(a, b \in L)$ if $a<b$ and if $a \leqslant c \leqslant b$ implies $c=a$ or $c=b$, for all $c \in L$. An element $p \in L$ is called an atom (respectively coatom) of $L$ if $0 \prec p$ (respectively $p \prec 1$ ). A lattice $L$ is called atomistic (respectively coatomistic) if every element of $L$ is a join of atoms (respectively meet of coatoms). For more details, see [6] section (5.2). If $L$ is a bounded lattice, we denote respectively by $L_{\vee}$ and $L_{\wedge}$ the two corresponding $(\vee, 0)$-semilattice and $(\wedge, 1)$-semilattice. It is not hard to verify that an element of the $(\vee, 0)$-semilattice (respectively 
$(\wedge, 1)$-semilattice) $L_{\vee}$ (respectively $\left.L_{\wedge}\right)$ is irreducible if and only if it is an atom (respectively coatom) of the $(\vee, 0)$-semilattice (respectively $(\wedge, 1)$ semilattice) $L_{\vee}$ (respectively $L_{\wedge}$ ). This allows us to define the notion of factorial and cofactorial lattices : A bounded lattice $L$ will be called factorial (respectively cofactorial) if the $(\vee, 0)$-semilattice $L_{\vee}$ (respectively $L_{\wedge}$ ) is factorial (respectively cofactorial).

For an arbitrary group $G$, we denote by $\mathcal{N}(G)$ the bounded lattice of normal subgroup of $G$. Recall that for every normal subgroups $H$ and $K$ of $G$, the meet and the join of $H$ and $K$ in $\mathcal{N}(G)$ are defined by

$$
H \wedge K=H \cap K \quad \text { and } \quad H \vee K=H K .
$$

Let denote respectively by $\mathcal{N}_{\wedge}(G)$ and $\mathcal{N}_{\vee}(G)$ the two corresponding $(\wedge, 1)$-semilattice and $(\vee, 0)$-semilattice of the bounded lattice $\mathcal{N}(G)$. Let's recall firstly some notion of group theory which the reader can be found in [4]. For a subgroups $H$ and $K$ of $G$, we write $H \leqslant K$ if $H$ is a subgroup of $K$. A subgroup $H$ of $G$ is called a minimal normal subgroup if $H$ is a normal subgroup of $G$ and if for every normal subgroup $N$ of $G, N \leqslant H$ implies that $N=H$ or $N$ is trivial. If $H$ is a subgroup of $G$, a complement of $H$ in $G$ is any subgroup $K$ of $G$ such that $G=H K$ and $H \cap K=\{1\}$. We call normal complement of $H$ in $G$ any complement of $H$ in $G$ which is normal in $G$. Note that if $H$ is a normal subgroup of $G$, then $H$ have a normal complement in $G$ if and only if $H$ is direct factor in $G$, that is $G=H \times K$ for some subgroup $K$ of $G$ which is necessarily normal. The group $G$ is called a $T$-group if for every subgroups $H$ and $K$ of $G$, if $H$ is normal in $K$ and $K$ is normal in $G$, then $H$ is normal in $G$. That is every subnormal subgroup of $G$ is normal. For every elements $x, y \in G$, $[x, y]=x y x^{-1} y^{-1}$ denotes the commutator of $x$ and $y$. If $X$ and $Y$ are subsets of $G$, we denote by $[X, Y]$ the subgroup of $G$ generated by the commutators $[x, y]$, with $x \in X$ and $y \in Y$. The commutator subgroup of $G$, denoted by $D(G)$, is defined to be $[G, G]$. The group $G$ is called perfect if $D(G)=G$ and it is called super-perfect if $[G, H]=H$ for every normal subgroup $H$ of $G$.

Proposition 1. Let $G$ be a group. Then the atoms of the idempotent monoid $\mathcal{N}_{\vee}(G)$ are exactly the minimal normal subgroups of $G$.

Proof. Let $H$ be a normal subgroup of $G$, with $H \neq\{1\}$. Suppose that $H$ is an atom of $\mathcal{N}_{\mathrm{V}}(G)$ and let's prove that $H$ is a minimal normal subgroup of $G$. Assume that $H$ is not a minimal normal subgroup, then there exist a non trivial normal subgroup $K$ of $G$ such that $K_{1}<H$. Denote by 
$K_{2}$ the subgroup of $G$ generated by $H \backslash K_{1}$. We can check easily that $K_{2}$ is a normal subgroup of $G$ contained in $H$, distinct to $K_{1}$ and that $H=K_{1} K_{2}$. Since $H$ is an atom of $\mathcal{N}_{\mathrm{V}}(G)$, then $K_{1}=\{1\}$ or $K_{2}=\{1\}$. But by hypothesis $K_{1} \neq\{1\}$, then $K_{2}=\{1\}$ which implies that $K_{1}=H$ which is impossible. Consquently, $H$ is a minimal normal subgroup of $G$. Conversely, assume that $H$ is a minimal normal subgroup of $G$. If $H_{1}$ and $H_{2}$ are two distinct normal subgroup of $G$ such that $H=H_{1} H_{2}$, then obviously $H_{1} \leqslant H$ and $H_{2} \leqslant H$ which implies by minimality of $H$ that $H_{1}=\{1\}$ or $H_{1}=H$ and $H_{2}=\{1\}$ or $H_{2}=H$. The case where $H_{1}=\{1\}$ and $H_{2}=\{1\}$ and likewise, the case where $H_{1}=H$ and $H_{2}=H$ are impossible because $H$ is not trivial and because $H_{1}$ and $H_{2}$ are distinct. The two other cases implies that $H_{1}=\{1\}$ or $H_{2}=\{1\}$. We deduce then that $H$ is an atom of $\mathcal{N}_{\mathrm{V}}(G)$.

\section{Remark 1.}

1) In the same way, we can check easily that the atoms of the idempotent monoid $\mathcal{N}_{\wedge}(G)$ are exactly the maximal normal subgroups of $G$.

2 ) It is clear that any simple normal subgroup of a given group $G$ is a minimal normal subgroup, but the converse is not true in general. Otherwise, for a T-group $G$, it is obviously true that any minimal normal subgroup of $G$ is simple.

Proposition 2. Let $G$ be a group and let $H_{1}, H_{2}, \ldots, H_{n}$ are simple normal subgroups of $G$. If $G=H_{1} H_{2} \ldots H_{n}$, then there exist some indices $i_{1}, \ldots, i_{k} \in\{1, \ldots, n\}$ such that

$$
G=H_{i_{1}} \times \cdots \times H_{i_{k}} .
$$

Proof. Let $I=\{1, \ldots, n\}$ and let $\mathcal{E}$ be the partially ordered set (ordered by inclusion) of non empty subsets $J$ of $I, J=\left\{j_{1}, \ldots, j_{r}\right\}$, such that $G=H_{j_{1}} \ldots H_{j_{r}}$. It is clear that $\mathcal{E}$ is non empty (it contain $I$ itself). Let $J=\left\{j_{1}, \ldots, j_{r}\right\}$ be an element of $\mathcal{E}$ of a minimal cardinal. Suppose that the product $G=H_{j_{1}} \ldots H_{j_{r}}$ is not direct. Then we have $H_{j_{\ell}} \cap$ $\left(H_{j_{1}} \ldots \widehat{H_{j_{\ell}}} \ldots H_{j_{r}}\right) \neq\left\{1_{G}\right\}$, for some index $j_{\ell} \in J$, where the hat means that the product is taken for all indices $j_{1}, \ldots, j_{r}$ except the index $j_{\ell}$. But since $H_{j_{\ell}}$ is simple, then we have $H_{j_{\ell}} \cap\left(H_{j_{1}} \ldots \widehat{H_{j_{\ell}}} \ldots H_{j_{r}}\right)=H_{j_{\ell}}$ which implies that $H_{j_{\ell}} \subset\left(H_{j_{1}} \ldots \widehat{H_{j_{\ell}}} \ldots H_{j_{r}}\right)$. Therefore we have $G=$ $H_{j_{1}} \ldots \widehat{H_{j_{\ell}}} \ldots H_{j_{r}}$ which is impossible.

Proposition 3 ([5], proposition (1.6.3)). Let $G=G_{1} \times \cdots \times G_{n}$ and $H$ be a normal subgroup of $G$. If $G_{1}, \ldots, G_{n}$ are non abelian simple groups, 
then there exists a subset $J=\left\{j_{1}, \ldots, j_{r}\right\} \subset\{1, \ldots, n\}$, such that

$$
H=G_{j_{1}} \times \cdots \times G_{j_{r}} .
$$

Proposition 4. Let $G$ be a group. If $G=G_{1} \times \cdots \times G_{n}$, where $G_{1}, \ldots, G_{n}$ are non abelian simple subgroups, then $G$ is super-perfect.

Proof. Let $H$ be a normal subgroup of $G$. By the previous proposition, we have $H=G_{i_{1}} \times \ldots G_{i_{r}}$ for some indices $i_{1}, \ldots, i_{r} \in\{1, \ldots, n\}$. By rearranging the indices, we can assume that $H=G_{1} \times \cdots \times G_{r}$. The subgroup $[G, H]$ is a normal subgroup of $G$, thus it is a normal subgroup of $H$. By a way of contraposition, assume that $[G, H]<H$ which implies that some factor $G_{i}$, for $1 \leqslant i \leqslant r$, is not appear in $[G, H]$. Say for example that $G_{r}$ is not appear in $[G, H]$. We have then $[G, H] \leqslant G_{1} \times \cdots \times G_{r-1}$. For every $x, y \in G_{r}$, we have $[x, y]=x y x^{-1} y^{-1} \in G_{r} \cap\left(G_{1} \ldots G_{r-1}\right)=\left\{1_{G}\right\}$ which implies that $G_{r}$ is abelain which is a contradiction. We deduce then that $[G, H]=H$ for every normal subgroup of $G$ and then $G$ is super-perfect.

\section{ND-groups and UND-groups}

A group $G$ is called an ND-group if every normal subgroup of $G$ has a normal complement. J. Weigold in [1], theorem (4.5), prove that a group is an ND-group if and only if it is the restricted direct product of simple groups. In order to characterize the groups $G$ for which the idempotent monoid $\mathcal{N}_{\mathrm{V}}(G)$ is factorial we need to introduce a subclass of the class of ND-groups. A group $G$ is called an UND-group if every normal subgroup of $G$ has a unique normal complement. If $H$ is a normal subgroup of an UND-group $G$, we denote by $H^{\perp}$ the unique normal complement of $H$ in $G$. Obviously, every UND-group is an ND-group but the converse is not true in general. For example, we let the reader to check that the Klein group $\mathbb{Z} / 2 \mathbb{Z} \times \mathbb{Z} / 2 \mathbb{Z}$ is an ND-group but it is not an UND-group.

The first thing what we can say about the ND-groups that they are in fact T-groups. Indeed, let $G$ be an ND-group and let $N$ be a normal subgroup of $G$. We will check that every normal subgroup of $N$ is normal in $G$. For that, take a normal subgroup $M$ of $N$. Since $G$ is an ND-group and $N$ is one of its normal subgroup, then $G=R \times N$ for some normal subgroup $R$ of $G$. For $m \in M$ and for $g=r n \in G$, with $n \in N$ and $r \in R$, we have $g m g^{-1}=r\left(n m n^{-1}\right) r^{-1}=n m n^{-1} \in M$, as $M$ is normal in $N$ and $[R, M]=\{1\}$. By the remark (2.3), we deduce then that for an ND-group $G$, the atoms of the monoid $\mathcal{N}_{V}(G)$ are exactly the simple 
normal subgroups of $G$. It has been proven, lemma (4.1) of [1], that every normal subgroup of an ND-group is an ND-group. In the following result we prove the same property for the UND-groups :

Lemma 1. Every normal subgroup of an UND-group is an UND-group.

Proof. Let $G$ be an UND-group and let $H$ be a normal subgroup of $G$. We will show that every normal subgroup of $H$ have a unique normal complement in $H$. Let then $K$ be a normal subgroup of $H$. As $G$ is a T-group, then $K$ is a normal subgroup of $G$. Therefore since $G$ is an UNDgroup, there exist a unique normal subgroup $L$ of $G$ such that $G=K \times L$. The subgroup $L \cap H$ is normal in $G$ and it is contained in $H$, hence it is a normal subgroup of $H$. We will show that $H=K \times(L \cap H)$. As $K$ and $L \cap H$ are normal subgroups of $H$, then $K(L \cap H)$ is a subgroup of $H$. Let $h \in H$, then $h=k l$ for some element $(k, l) \in K \times L$ as $G=K \times L$. We have $l=h k^{-1} \in H$, then $l \in L \cap H$ which implies that $h=k l \in K(L \cap H)$. Hence $H \subset K(L \cap H)$ and then $H=K(L \cap H)$. Moreover, we have clearly $K \cap(L \cap H)=\{1\}$, as $K \cap L=\{1\}$, which implies that $H=K \times(L \cap H)$. We deduce then that $L \cap H$ is a normal complement of $K$ in $H$. Suppose that $V$ is another normal complement of $K$ in $H$, that is $V$ is a normal subgroup of $H$ and $H=K \times V$. We will prove that $V=L \cap H$. As $G$ is an UND-group and since $H$ is normal in $G$, then there exist a unique normal subgroup $H^{\prime}$ of $G$ such that $G=H \times H^{\prime}$. But we have $H=K \times(L \cap H)$ and $H=K \times V$, then we have $K \times\left((L \cap H) \times H^{\prime}\right)=G$ and $K \times\left(V \times H^{\prime}\right)=G$. This implies that $(L \cap H) \times H^{\prime}$ and $V \times H^{\prime}$ are two normal complement of $K$ in $G$. Thus we have the equality $(L \cap H) \times H^{\prime}=V \times H^{\prime}$. Let's prove now that $V=L \cap H$. Let $v \in V$, then $v=x y$ for some element $(x, y) \in(L \cap H) \times H^{\prime}$ because $V$ is a subgroup of $(L \cap H) \times H^{\prime}$. But since $v \in V \subset H$ and since $x \in L \cap H \subset H$, then $x^{-1} v \in H$. Or $x^{-1} v=y \in H^{\prime}$, then $x^{-1} v \in H \cap H^{\prime}=\{1\}$ which implies that $v=x \in L \cap H$. We deduce then that $V \subset L \cap H$. Likewise, if $\ell \in L \cap H$, then $\ell=a b$ for some element $(a, b) \in V \times H^{\prime}$. We have $\ell \in L \cap H \subset H$ and $a \in V \subset H$, then $a^{-1} \ell \in H$. But we have too $a^{-1} \ell=b \in H^{\prime}$, then $a^{-1} \ell \in H \cap H^{\prime}=\{1\}$ and then $\ell=a \in V$. We deduce then that $L \cap H \subset V$ and consequently the equality $V=L \cap H$ is obtained which proves that the normal subgroup $H$ of $G$ is an UND-group.

Lemma 2. Let $G$ be an UND-group and let $H$ be a simple normal subgroup of $G$. Then for every normal subgroups $K_{1}$ and $K_{2}$ of $G$, with $K_{1} \cap K_{2}=$ $\{1\}$, if $H \leqslant K_{1} \times K_{2}$ then $H \leqslant K_{1}$ or $H \leqslant K_{2}$. 
Proof. Let $K_{1}$ and $K_{2}$ two normal subgroups of $G$, with $K_{1} \cap K_{2}=\{1\}$, such that $H$ is a subgroup of $K_{1} \times K_{2}$. Denote by $S$ the subgroup $H \cap K_{1}$. Since $S$ is a normal subgroup of $H$, then $S=\{1\}$ or $S=H$. If $S=H$, then $H \leqslant K_{1}$. Otherwise, $H \cap K_{1}=\{1\}$ which implies that $K_{1} H=K_{1} \times H$. As $H$ and $K_{1}$ are two normal subgroups of $K_{1} \times K_{2}$, then $K_{1} \times H$ is a normal subgroup of $K_{1} \times K_{2}$. But since $G$ is an UND-group, then by the proposition (3.1) the normal subgroup $K_{1} \times K_{2}$ of $G$ is also an UND-group. There exist then a normal subgroup $M$ of $K_{1} \times K_{2}$, which is normal in $G$ as $G$ is a $T$-group, such that $K_{1} \times(H \times M)=\left(K_{1} \times H\right) \times M=K_{1} \times K_{2}$. The previous equality implies that the subgroups $H \times M$ and $K_{2}$ are two normal complement of $K_{1}$ in the group $K_{1} \times K_{2}$. Then we have $H \times M=K_{2}$ which implies that $H$ is a subgroup of $K_{2}$. We deduce then that $H \leqslant K_{1}$ or $H \leqslant K_{2}$.

Corollary 1. Let $G$ be an UND-group and let $H$ be a simple normal subgroup of $G$. Then for every normal subgroups $K_{1}, \ldots, K_{n}$ of $G$, if

$$
H \leqslant K_{1} \times K_{2} \times \cdots \times K_{n}
$$

then there exist a unique $i \in\{1, \ldots, n\}$ such that $H \leqslant K_{i}$.

Proof. Immediate by induction.

Corollary 2. Let $G$ be an UND-group and let $H$ be a simple normal subgroup of $G$. Then for every simple normal subgroups $K_{1}, \ldots, K_{n}$ of $G$, if

$$
H \leqslant K_{1} \times K_{2} \times \cdots \times K_{n}
$$

then there exist a unique $i \in\{1, \ldots, n\}$ such that $H=K_{i}$.

Proof. Immediately follows from the previous corollary.

Lemma 3. Let $G$ be a group. If $G$ is an UND-group, then for every pairwise distinct simple normal subgroups $K_{1}, \ldots, K_{n}$ of $G$, we have

$$
K_{1} K_{2} \ldots K_{n}=K_{1} \times K_{2} \times \cdots \times K_{n}
$$

Proof. Assume to the contrary that the product $K_{1} K_{2} \ldots K_{n}$ is not a direct product, then there exist some index $j \in\{1,2, \ldots, n\}$ such that $K_{j} \cap$ $\left(K_{1} \ldots \widehat{K}_{j} \ldots K_{n}\right) \neq\{1\}$. Since $K_{j}$ is simple and as $K_{j} \cap\left(K_{1} \ldots \widehat{K}_{j} \ldots K_{n}\right)$ is a non trivial normal subgroup of $K_{j}$, then $K_{j} \cap\left(K_{1} \ldots \widehat{K}_{j} \ldots K_{n}\right)=K_{j}$ which implies that $K_{j} \subset K_{1} \ldots \widehat{K}_{j} \ldots K_{n}$. By the proposition (2.4) we 
can extract from the product $K_{1} \ldots \widehat{K}_{j} \ldots K_{n}$ a direct product, that is for some indices $i_{1}, \ldots, i_{k} \in\{1,2, \ldots, n\} \backslash\{j\}$ we have

$$
K_{1} \ldots \widehat{K}_{j} \ldots K_{n}=K_{i_{1}} \times \cdots \times K_{i_{k}} .
$$

Therefore the simple normal subgroup $K_{j}$ of $G$ is in fact a simple normal subgroup of the direct product $K_{i_{1}} \times \cdots \times K_{i_{k}}$. Then by the previous corollary $K_{j}=K_{i_{\ell}}$ for some index $i_{\ell} \in\{1,2, \ldots, n\} \backslash\{j\}$ which is impossible since the subgroups $K_{1}, K_{2}, \ldots, K_{n}$ are pairwise distinct.

Recall that a group $G$ is called of finite length if it satisfies both ascending and descending chain conditions on normal subgroups. We conclude this section by proving that an ND-group is of finite length if and only if it is the direct product of a finite number of simple groups. We will use this fact in the next section.

Lemma 4. Let $G$ be an ND-group, $H_{0}$ be a normal subgroup of $G$ and $K_{0}$ be a normal complement of $H_{0}$ in $G$. For every normal subgroup $H_{1}$ of $G$, if $H_{1} \leqslant H_{0}$, then there exist a normal complement $K_{1}$ of $H_{1}$ in $G$ such that $K_{0} \leqslant K_{1}$.

Proof. Let $H_{1}$ be a normal subgroup of $G$ such that $H_{1} \leqslant H_{0}$. Since $H_{0}$ is a normal subgroup of the ND-group $G$, then by lemma (4.1) of [1], $H_{0}$ is also an ND-group. Therefore we have $H_{0}=H_{1} \times L$ for some normal subgroup $L$ of $H_{0}$ which is in fact a normal subgroup of $G$ since $G$ in an ND-group and in particular a $T$-group. If we denote by $K_{1}$ the normal subgroup $L K_{0}$, then it is clear that $K_{0} \leqslant K_{1}$. We will prove that $K_{1}$ is a normal complement of $H_{1}$ in $G$. We have $H_{1} K_{1}=H_{1}\left(L K_{0}\right)=\left(H_{1} L\right) K_{0}=H_{0} K_{0}=G$. Furthermore, if $x \in H_{1} \cap K_{1}$ then $x \in H_{0}$ since $x \in H_{1} \leqslant H_{0}$ and $x=u v$ for some element $(u, v) \in L \times K_{0}$ as $x \in K_{1}=L K_{0}$. As $L \leqslant H_{0}$, then $u \in H_{0}$ and therefore $u^{-1} x=v \in H_{0} \cap K_{0}=\{1\}$. Thus we have $x=u \in L$, but $x \in H_{1}$, then $x \in H_{1} \cap L=\{1\}$ which implies that $x=1$. We have then $H_{1} \cap K_{1}=\{1\}$ and consequently $K_{1}$ is a normal complement of $H_{1}$ in $G$.

Corollary 3. Let $G$ be an ND-group. Then for every decreasing sequence $\left(H_{n}\right)_{n \in \mathbb{N}}$ of normal subgroups of $G$, there exist a sequence $\left(K_{n}\right)_{n \in \mathbb{N}}$ of normal subgroups of $G$ such that :

1) for every $n \in \mathbb{N}, K_{n}$ is a normal complement of $H_{n}$ in $G$,

2) the sequence $\left(K_{n}\right)_{n \in \mathbb{N}}$ is increasing.

Proof. Using the previous lemma, we can easily construct a such sequence by induction. 
Corollary 4. Let $G$ be an ND-group. Then $G$ satisfy the descending chain condition on normal subgroups if and only if it satisfy the ascending chain condition on normal subgroups.

Proof. Suppose that $G$ have the ascending chain condition on normal subgroups. Let $\left(H_{n}\right)_{n \in \mathbb{N}}$ be a decreasing sequence of normal subgroups of $G$. By the previous corollary, there exist an increasing sequence $\left(K_{n}\right)_{n \in \mathbb{N}}$ of normal subgroups of $G$ such that, for every $n \in \mathbb{N}, K_{n}$ is a normal complement of $H_{n}$ in $G$. Since $G$ have the ascending chain condition, then there exist an integer $n_{0} \in \mathbb{N}$ such that $K_{n}=K_{n_{0}}$, for every $n \geqslant n_{0}$. We will show that, for every $n \geqslant n_{0}, H_{n}=H_{n_{0}}$. Since the sequence $\left(H_{n}\right)_{n \in \mathbb{N}}$ is decreasing, then $H_{n} \leqslant H_{n_{0}}$ for every $n \geqslant n_{0}$. Let $n \geqslant n_{0}$ and let $x \in H_{n_{0}}$. We have $G=H_{n} \times K_{n}$, but $K_{n}=K_{n_{0}}$, then we have $G=H_{n} \times K_{n_{0}}$. Thus we have $x=a b$, for some element $(a, b) \in H_{n} \times K_{n_{0}}$ which implies that $a^{-1} x=b \in K_{n_{0}}$. But since $a \in H_{n} \leqslant H_{n_{0}}$, then $a \in H_{n_{0}}$ which implies that $a^{-1} x \in H_{n_{0}}$. Therefore $a^{-1} x \in H_{n_{0}} \cap K_{n_{0}}=\{1\}$ and then $x=a \in H_{n}$. We deduce then that $H_{n_{0}} \leqslant H_{n}$. Consequently, for every $n \geqslant n_{0}, H_{n}=H_{n_{0}}$. Thus $G$ have descending chain condition on normal subgroups. By the same way, we can prove the other implication.

By the previous corollary, clearly an ND-group is of finite length if it satisfies the ascending or the descending chain condition on normal subgroups.

Proposition 5. Let $G$ be an ND-group. Then $G$ is of finite length if and only if $G$ is a direct product of a finite number of simple groups.

Proof. Suppose that $G$ is of finite length. By the Krull-Schmidt theorem, theorem (3.3) page 83 of [4], the group $G$ is then the direct product of a finite number of indecomposable subgroups, say $G=G_{1} \times G_{2} \times \cdots \times G_{r}$ where $G_{1}, \ldots, G_{r}$ are indecomposable subgroups of $G$. Every factor $G_{i}$, for $1 \leqslant i \leqslant r$, is a normal subgroup of $G$, thus $G_{i}$ is an ND-group. But obviously an indecomposable ND-group is simple. Therefore all the factor $G_{1}, \ldots, G_{r}$ are simple and hence $G$ is a direct product of a finite number of simple groups. The converse is immediate.

\section{Main results}

Theorem 1. Let $G$ be group. Then the commutative idempotent monoid $\mathcal{N}_{\vee}(G)$ is atomic if and only if the group $G$ is an ND-group with finite length. 
Proof. Suppose that $\mathcal{N}_{\vee}(G)$ is atomic. Then every normal subgroup of $G$ can be expressed as product of pairwise distinct simple normal subgroups. In particular, there exist some pairwise distinct simple normal subgroups $G_{1}, G_{2}, \ldots, G_{n}$ such that $G=G_{1} \ldots G_{n}$. By the proposition (2.4), we can assume that $G=G_{1} \times \cdots \times G_{n}$. Let $H$ be a normal subgroup of $G$. We will show that $H$ is a direct factor in $G$. There are three cases : For the first case, assume that all the simple normal subgroups $G_{i}$ are non abelian. As $H$ is a normal subgroup of $G$, by the proposition (2.5) we have $H=G_{i_{1}} \times \cdots \times G_{i_{s}}$ for some subset $J=\left\{i_{1}, \ldots, i_{s}\right\}$ of $I=\{1, \ldots, n\}$. In this case, it is clear that $G=H \times K$ where $K$ is the product of the $G_{i}$ 's for $i \in I \backslash J$. For the second case, assume that some but not all of the subgroups $G_{i}$ are abelian, say for example that $G_{1}, \ldots, G_{r}$ are abelian and $G_{r+1}, \ldots, G_{n}$ are non abelian, where $1<r<n$. If we put $A=G_{1} \times \cdots \times G_{r}$ and $K=G_{r+1} \times \cdots \times G_{n}$, then we have $G=A \times K$. Since $K=G_{r+1} \times \cdots \times G_{n}$, where $G_{r+1}, \ldots, G_{r}$ are non abelian simple groups, then by the proposition (2.6) the group $K$ is super-perfect. Hence $H$ is a normal subgroup of $G=A \times K$ with $K$ is super-perfect. Then by the theorem 1 of [2], page 155, $H=B \times L$ for some normal subgroup $B$ of $A$ and some normal subgroup $L$ of $K$. By the first case, since $L$ is normal in $K$ and as $K$ is the direct product of simple non abelian groups, then $K=L \times M$ for some normal subgroup $M$ of $K$. Moreover, as $A$ is the direct product of simple abelian groups we know that every subgroup of $A$ is a direct factor in $A$. Then we have $A=B \times C$ for some subgroup $C$ of $A$. Note that the subgroup $M$ (resp. $C$ ) is in fact normal in $G$ since it is a normal subgroup of a direct factor of $G$. If we denote $H^{\prime}=C \times M$ then clearly $H^{\prime}$ is a normal complement of $H$ is $G$. We deduce then that $G$ is an ND-group. For the last case, all the simple subgroups $G_{i}$, for $1 \leqslant i \leqslant n$, are assumed to be abelian. In this case, the group $G$ is then an abelian ND-group and the result in this case is obvious. Conversely, suppose that $G$ is an ND-group with finite length and let's prove that the idempotent monoid $\mathcal{N}_{\vee}(G)$ is atomic. Let $H$ be a normal subgroup of $G$. Since $G$ is an ND-group, then $H$ is an ND-group and as $G$ is of finite length then $H$ is also of finite length. Therefore, by the proposition (3.9) $H$ is the direct product of a finite number of simple normal subgroups, which are normal in $G$ as $G$ is a T-group. Thus $H$ is a product of a finite number of pairwise simple normal subgroups of $G$ and the fact that the idempotent monoid $\mathcal{N}_{\vee}(G)$ is atomic is proved.

Theorem 2. Let $G$ be a group. Then the idempotent monoid $\mathcal{N}_{\vee}(G)$ is factorial if and only if the group $G$ is an UND-groups with finite length. 
Proof. Suppose that $G$ is an UND-group with finite length. By the theorem (4.1), the monoid $\mathcal{N}_{\vee}(G)$ is in particular atomic. Let $H_{1}, \ldots, H_{n}$ a pairwise distinct simple normal subgroups and $K_{1}, \ldots, K_{m}$ a pairwise distinct simple normal subgroups of $G$ such that $H_{1} \ldots H_{n}=K_{1} \ldots K_{m}$. We must prove that $n=m$ and there exist a permutation $\sigma \in S_{n}$ such that, for every $i \in\{1, \ldots, n\}, K_{i}=H_{\sigma(i)}$. Since $G$ is an UND-group, then by the lemma (3.5) we have

$$
H_{1} \ldots H_{n}=H_{1} \times \cdots \times H_{n} \quad \text { and } \quad K_{1} \ldots K_{m}=K_{1} \times \cdots \times K_{m} .
$$

Let's show firstly that we have necessarily $n=m$. Suppose that $n \neq m$, for example take $n<m$. Let $i \in\{1, \ldots, n\}$, since $H_{i}$ is a subgroup of the direct product $K_{1} \times \cdots \times K_{m}$ then by the corollary (3.4), we have $H_{i}=K_{j_{i}}$ for a unique index $j_{i} \in\{1, \ldots, m\}$. Since the subgroups $H_{1}, \ldots, H_{n}$ are pairwise distinct, then the indices $j_{1}, \ldots, j_{n} \in\{1, \ldots, m\}$ are pairwise distinct. But as $n<m$, then there exist an index $\ell \in\{1, \ldots, m\}$ such that $\ell \notin\left\{j_{1}, \ldots, j_{n}\right\}$. Now $K_{\ell}$ is a subgroup of $H_{1} \times \cdots \times H_{n}$, then by the corollary (3.4) we have $K_{\ell}=H_{k}$ for a unique index $k \in\{1, \ldots, n\}$, but $H_{k}=K_{j_{k}}$. Then we have $K_{\ell}=K_{j_{k}}$ which implies that $\ell=j_{k}$ as the subgroups $K_{1}, \ldots, K_{m}$ are pairwise distinct. We have then $\ell \in\left\{j_{1}, \ldots, j_{n}\right\}$ which is a contradiction. We deduce then that $n=m$. We will prove now that there exist a permutation $\sigma \in S_{n}$ such that, for every $i \in\{1, \ldots, n\}$, $K_{i}=H_{\sigma(i)}$. By the same way as the previous proof, clearly for every $i \in\{1, \ldots, n\}$ there exist a unique index $\sigma(i) \in\{1, \ldots, n\}$ such that $K_{i}=H_{\sigma(i)}$. We must show that the map $\sigma$ is in fact injective, but this is clearly true since the subgroups $K_{1}, \ldots, K_{n}$ are pairwise distinct. We deduce then that the idempotent monoid $\mathcal{N}_{\mathrm{V}}(G)$ is factorial. Conversely, suppose that $\mathcal{N}_{\vee}(G)$ is factorial and let's prove that the group $G$ is an UND-group with finite length. As $\mathcal{N}_{\vee}(G)$ is factorial, it is in particular atomic and then by the previous theorem the group $G$ is an ND-group with finite length. Let $H$ be a normal subgroup of $G$ and let $K, L$ two normal subgroups of $G$ such that $H \times K=G$ and $H \times L=G$. We will show that $K=L$. Since $\mathcal{N}_{\mathrm{V}}(G)$ is atomic, then $H=H_{1} \ldots H_{r}$ for some pairwise distinct simple normal subgroups $H_{1}, \ldots, H_{r}, K=K_{r+1} \ldots K_{r+k}$ for some pairwise distinct simple normal subgroups $K_{r+1}, \ldots, K_{r+k}$ and $L=L_{r+1} \ldots L_{r+\ell}$ for some pairwise distinct simple normal subgroups $L_{r+1}, \ldots, L_{r+\ell}$. If we put, for $i \in\{1, \ldots, r\}, K_{i}=L_{i}=H_{i}$, then we have

$$
K_{1} \ldots K_{r} K_{r+1} \ldots K_{r+k}=L_{1} \ldots L_{r} L_{r+1} \ldots L_{r+\ell}
$$

Since $H \cap K=\{1\}$ and $H \cap L=\{1\}$, then the normal simple subgroups $K_{1}, \ldots, K_{r+k}$ are in fact pairwise distinct and the same holds for the 
normal simple subgroups $L_{1}, \ldots, L_{r+\ell}$. As $\mathcal{N}_{\vee}(G)$ is factorial, then $r+k=$ $r+\ell$ which implies that $k=\ell$ and there exist a permutation $\sigma \in S_{r+k}$ such that, for every $i \in\{1, \ldots, r+k\}, L_{i}=K_{\sigma(i)}$. Obviously, for every $i \in\{r+1, \ldots, r+k\}, \sigma(i) \in\{r+1, \ldots, r+k\}$, indeed if there exist $i_{0} \in$ $\{r+1, \ldots, r+k\}$ such that $\sigma\left(i_{0}\right) \in\{1, \ldots, r\}$ then $L_{i_{0}}=K_{\sigma\left(i_{0}\right)}=H_{\sigma\left(i_{0}\right)}$ which is impossible as $H \cap L=\{1\}$. Consequently we have

$$
L=L_{r+1} \ldots L_{r+k}=K_{\sigma(r+1)} \ldots K_{\sigma(r+k)}=K .
$$

We deduce then that the group $G$ is an UND-group and we have already proved that $G$ is of finite length.

Note that in an UND-group $G$, it is easy to check that a non trivial and proper normal subgroup $H$ of $G$ is an atom of the idempotent monoid $\mathcal{N}_{\vee}(G)$ if and only if $H^{\perp}$ is an atom of the idempotent monoid $\mathcal{N}_{\wedge}(G)$. Furthermore, obviously for every normal subgroups $H_{1}$ and $H_{2}$ of $G$, we have

$$
\left(H_{1} \vee H_{2}\right)^{\perp}=H_{1}^{\perp} \wedge H_{2}^{\perp} \quad \text { and } \quad\left(H_{1} \wedge H_{2}\right)^{\perp}=H_{1}^{\perp} \vee H_{2}^{\perp} .
$$

Using this simple facts, we can show easily that for an arbitrary group $G$, if the idempotent monoid $\mathcal{N}_{\vee}(G)$ is factorial then the idempotent monoid $\mathcal{N}_{\wedge}(G)$ is factorial.

Recall that a partial commutative monoid, see [3] definition (2.1.1), is a structure $(P, \oplus, 0)$, where $P$ is a set, $0 \in P$, and $\oplus$ is a partial binary operation on $P$ satisfying the following properties, for all $x, y, z \in P$ :

(P1) Associativity : $x \oplus(y \oplus z)$ is defined iff $(x \oplus y) \oplus z$ is defined, and then the two values are equal.

(P2) Commutativity : $x \oplus y$ is defined iff $y \oplus x$ is defined, and then the two values are equal.

(P3) Zero element : $x \oplus 0$ is defined with values $x$.

To any bounded lattice $(L, \wedge, \vee)$ we can associate a structure of partial monoid $P_{L}$ as follows : the underlaying set of $P_{L}$ is $L$ and the partial binary operation of $P_{L}$ is defined by, for all $x, y \in L$,

$x \oplus y$ is defined only when $x \wedge y=0$ in case $x \oplus y=x \vee y$.

A partial commutative monoid $(P, \oplus, 0)$ is a partial refinement monoid (or have the refinement property), see [3] definition (2.2.1), if for all $x_{0}, x_{1}, y_{0}, y_{1} \in P$ with $x_{0} \oplus x_{1}=y_{0} \oplus y_{1}$, there are elements $c_{i, j} \in P$, for $i, j \in\{0,1\}$, such that $x_{i}=c_{i, 0} \oplus c_{i, 1}$ and $y_{j}=c_{0, j} \oplus c_{1, j}$ for every $i, j \in\{0,1\}$. If $G$ is a group, we denote by $\mathcal{N}_{\text {par }}(G)$ the partial commutative 
monoid associated to the bounded lattice of normal subgroup of $G$. We have then the following result:

Theorem 3. Let $G$ be an ND-group with finite length. Then $G$ is an $U N D$-group if and only if the partial commutative monoid $\mathcal{N}_{\text {par }}(G)$ has the refinement property.

Proof. Suppose that the partial commutative monoid $\mathcal{N}_{\text {par }}(G)$ has the refinement property and let's prove that $G$ is an UND-group. Let $H$ be a normal subgroup of $G$ and let $K_{0}, K_{1}$ two normal subgroup of $G$ such that $G=H \times K_{0}$ and $G=H \times K_{1}$, that is $K_{0}$ and $K_{1}$ are two normal complement of $H$ in $G$. Since $\mathcal{N}_{\text {par }}(G)$ has the refinement property and as $H \times K_{0}=H \times K_{1}$, then there exist normal subgroups $C_{i, j}$ of $G$, for $i, j \in\{0,1\}$, such that $H=C_{0,0} \times C_{0,1}, H=C_{0,0} \times C_{1,0}, K_{0}=C_{1,0} \times C_{1,1}$ and $K_{1}=C_{0,1} \times C_{1,1}$. It is clear that the subgroup $C_{1,0}$ is contained in $H$ and in $K_{1}$ which implies that $C_{1,0}$ is contained in $H \cap K_{1}=\{1\}$ and hence $C_{1,0}=\{1\}$. Likewise, the subgroup $C_{0,1}$ is contained in $H$ and in $K_{2}$ which implies that $C_{0,1}=\{1\}$. Therefore we have

$$
K_{0}=C_{1,0} \times C_{1,1}=C_{1,1}=C_{0,1} \times C_{1,1}=K_{1} .
$$

Consequently, the group $G$ is an UND-group. Conversely, suppose that the group $G$ is an UND-group and let's prove that the partial commutative monoid $\mathcal{N}_{\text {par }}(G)$ has the refinement property. Let $H_{0}, H_{1}, K_{0}, K_{1}$ be normal subgroups of $G$ such that $H_{0} \times H_{1}=K_{0} \times K_{1}$. Since $G$ is an UND-group with finite length then by theorem 2 , the idempotent monoid $\mathcal{N}_{\vee}(G)$ is factorial. Therefore, for $i \in\{0,1\}$, there exist a family of pairwise distinct simple normal subgroups $\left(H_{i, j}\right)_{0 \leqslant j \leqslant p_{i}}$ such that

$$
H_{i}=H_{i, 0} H_{i, 1} \ldots H_{i, p_{i}}, \quad i \in\{0,1\}
$$

and, for $j \in\{0,1\}$, there exist a family of pairwise distinct simple normal subgroups $\left(K_{i, j}\right)_{0 \leqslant i \leqslant q_{i}}$ such that

$$
K_{j}=K_{0, j} K_{1, j} \ldots K_{q_{j}, j}, \quad j \in\{0,1\} .
$$

By the corollary (4.5), all the previous products are in fact direct products. Hence we have

$H_{0,0} \times \cdots \times H_{0, p_{0}} \times H_{1,0} \times \cdots \times H_{1, p_{1}}=K_{0,0} \times \cdots \times K_{q_{0}, 0} \times K_{0,1} \times \cdots \times K_{q_{1}, 1}$.

For $i, j \in\{0,1\}$, put $C_{i, j}=H_{i} \cap K_{j}$. The subgroups $C_{i, j}$ are clearly normal in $G$ and we will show that we have the refinement matrix

\begin{tabular}{c|cc} 
& $K_{0}$ & $K_{1}$ \\
\hline$H_{0}$ & $C_{0,0}$ & $C_{0,1}$ \\
$H_{1}$ & $C_{1,0}$ & $C_{1,1}$
\end{tabular}


that is $H_{i}=C_{i, 0} \times C_{i, 1}$ for $0 \leqslant i \leqslant 1$ and $K_{j}=C_{0, j} \times C_{1, j}$ for $0 \leqslant j \leqslant 1$. We will show only that $H_{0}=C_{0,0} \times C_{0,1}$, the other equalities are proved in the same way. Since $C_{0,0}$ and $C_{0,1}$ are normal subgroups of $H_{0}$, then their product $C_{0,0} C_{0,1}$ is a subgroup of $H_{0}$. For the other inclusion, let $j \in\left\{0, \ldots, p_{0}\right\}$. As the simple normal subgroup $H_{0, j}$ is a subgroup of $H_{0} \times H_{1}$, and in particular a subgroup of $K_{0} \times K_{1}$, then by the corollary (4.4) we have $H_{0, j}=K_{i_{j}, 0}$ for some index $i_{j} \in\left\{0, \ldots, q_{0}\right\}$ or $H_{0, j}=K_{i_{j}, 1}$ for some index $i_{j} \in\left\{0, \ldots, q_{1}\right\}$. If $H_{0, j}=K_{i_{j}, 0}$ then obviously $H_{0, j}$ is a subgroup of $H_{0} \cap K_{0}$ and likewise if $H_{0, j}=K_{i_{j}, 1}$ then $H_{0, j}$ is a subgroup of $H_{0} \cap K_{1}$. Consequently, for every $j \in\left\{0, \ldots, p_{0}\right\}, H_{0, j}$ is a subgroup of $\left(H_{0} \cap K_{0}\right)\left(H_{0} \cap K_{1}\right)=C_{0,0} C_{0,1}$ and so $H_{0} \leqslant C_{0,0} C_{0,1}$. We deduce then that $H_{0}=C_{0,0} C_{0,1}$, but as $C_{0,0} \leqslant K_{0}$ and $C_{0,1} \leqslant K_{1}$, then $H_{0}=C_{0,0} \times C_{0,1}$. Consequently, the partial monoid $\mathcal{N}_{\text {par }}(G)$ has the refinement property.

\section{References}

[1] J. Weigold, "On direct factor in groups", J. London Math. Soc. 35 (1960), 310-320.

[2] M. D. Miller, "On the lattice of normal subgroups of a direct product", Pacific Jounrnal of mathematics. vol. 60, No 2, (1975).

[3] F. Wehrung, "Refinement monoids, equidecomposability types, and Boolean inverse semigroups". Lecture Notes in Mathematics, Springer Verlag, vol 2188 (2017).

[4] Thomas W. Hungerford, Algebra, 1th edn. Springer, New York (1980)

[5] Hans Kurzweil and Bernd Stellmacher, The Theory of Finite Groups An Introduction. Springer, New York (2004)

[6] B. A. Davey and H. A. Priestley, Introduction to Lattices and Order. Cambridge university press (1990)

[7] R. Schmidt, Subgroup lattices of groups. de Gruyter Expositions in Mathematics 14 (1994).

\section{CONTACT INFORMATION}

Anis Rajhi Mathematics Department, Faculty of Sciences and Humanities in Dawadmi, Shaqra University, 11911, Saudi Arabia;

Quantitative Methods Department, Higher business School, University of Manouba, Manouba 2010, Tunisia

E-Mail(s): anis.rajhi@math.univ-poitiers.fr

Received by the editors: 09.10.2018

and in final form 08.12.2020. 